\title{
Effects of dipeptidyl peptidase IV on the satiety actions of peptide YY
}

\author{
S. Unniappan - C. H. S. McIntosh • H.-U. Demuth • \\ U. Heiser $\cdot$ R. Wolf $\cdot$ T. J. Kieffer
}

Received: 22 February 2006 / Accepted: 6 April 2006 / Published online: 27 June 2006

(C) Springer-Verlag 2006

\begin{abstract}
Aims/hypothesis Dipeptidyl peptidase IV (DP IV) inhibitors are currently being developed to prolong the biological activity of insulinotropic peptides as a novel approach in the treatment of diabetes. We hypothesised that DP IV inhibition could attenuate the satiety actions of peptide YY (PYY) by altering the conversion of PYY(1-36) to PYY(3-36).

Materials and methods The effects of PYY delivered by osmotic mini-pumps were assessed in rats treated with a DP IV inhibitor and in a rat model deficient in DP IV.

Results Pharmacological levels of total PYY were found in the circulation after the exogenous administration of PYY (3-36). While both PYY(1-36) and PYY(3-36) reduced food intake in normal rats, PYY (1-36) was ineffective in rats deficient in DP IV. When re-fed after a 24-h fast, DP IV-deficient rats exhibited higher food intake and weight gain than normal rats. Moreover, unlike controls, there was no postprandial increase in PYY levels in DP IVdeficient rats. Despite these findings, administration of a DP IV inhibitor, Pro-boroPro, did not alter the acute

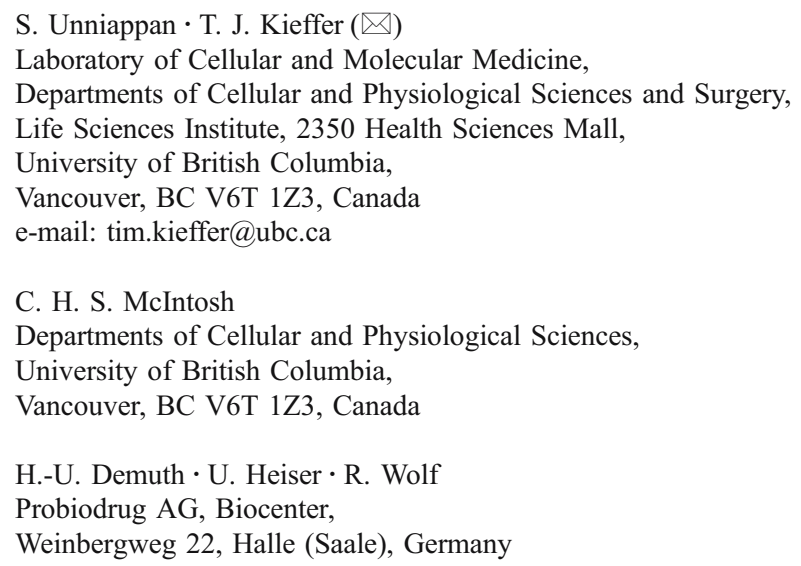

anorectic effects of exogenous PYY(1-36) in normal rats. This could be the result of the protection of other appetite regulatory peptides or the generation of PYY(3-36) by remaining DP IV activity or other dipeptidyl peptidases. Conclusions/interpretation Although DP IV inhibition with Pro-boroPro attenuated the generation of PYY(3-36), our results indicate that short-term DP IV inhibition does not eliminate the satiety actions of exogenously administered PYY (1-36) at the doses tested.

Keywords Body weight - Dipeptidyl peptidase IV.

Energy balance $\cdot$ Food intake $\cdot$ Gut $\cdot$ Hormone .

Osmotic mini-pumps · Peptide YY Pro-boroPro

\author{
Abbreviations \\ DP IV dipeptidyl peptidase IV \\ GIP glucose-dependent insulinotropic polypeptide \\ GLP-1 glucagon-like peptide-1 \\ MALDI matrix-assisted laser desorption/ionisation \\ PYY peptide YY \\ TOF time-of-flight
}

\section{Introduction}

Dipeptidyl peptidase IV (DP IV) is an aminopeptidase, which is present in both tissues and the circulation, and which cleaves dipeptides from the $\mathrm{N}$-terminal region of polypeptides with a strong preference for proline or alanine at the second position [1]. For example, DP IV rapidly cleaves glucose-dependent insulinotropic polypeptide (GIP) and glucagon-like peptide-1 (GLP-1), a process that eliminates their otherwise potent insulinotropic activities [2]. Two approaches are being actively pursued to take advantage of the antidiabetic effects of these incretin 
hormones. Analogues are being developed that are resistant to inactivation by DP IV, and inhibitors of DP IV are under investigation as a means of prolonging the biological activity of endogenous GIP and GLP-1 [3-5]. Several additional endocrine and neural factors are substrates of DP IV, including neuropeptide $\mathrm{Y}$, growth hormonereleasing factor, pituitary adenylate cyclase-activating polypeptide, gastrin-releasing peptide and peptide YY (PYY) [1, 6], raising the possibility that a lack of specificity of DP IV inhibitors might limit their use in treatment of diabetes.

PYY is a 36 amino acid peptide hormone released along with GLP-1 from L-cells of the distal intestine during meals [7]. DP IV cleaves the Tyr-Pro dipeptide from the N-terminus of PYY(1-36) to produce PYY(3-36), a second biologically active form of PYY [8-11]. Physiological functions regulated by PYY include glomerular filtration [12], vasoconstriction [13], gastric emptying [14], gastric acid secretion [15], secretion from the exocrine pancreas [16] and food intake [17]. Intracerebroventricular or i.p. injections [18-21] and s.c. or i.v. infusion [22-24] of PYY (3-36) in rodents, along with i.m. injections in rhesus monkeys [25] and i.v. infusion in lean and obese humans [26], have been shown to inhibit food intake. PYY(3-36) administration also inhibits weight gain in rodents $[18,23]$. The satiety actions of PYY(3-36) appear to be mediated by the Y2-receptor present in the hypothalamic arcuate nucleus $[17,27]$; thus satiety actions of PYY(3-36) are absent in Y2-receptor knock-out mice [18, 19]. However, there is some evidence to indicate a vagally mediated mechanism of peripheral effects of PYY [21, 28], although this remains controversial [20]. Intravenous infusion of PYY(1-36) also inhibits food intake in rats [29], probably as a result of DP IV-mediated conversion to PYY(3-36). In striking contrast, intracerebroventricular or paraventricular injections of PYY(1-36) stimulate food intake [30, 31], probably acting via hypothalamic $\mathrm{Y} 1$ and $\mathrm{Y} 5$ receptors [32].

Diminished DP IV activity would be predicted to attenuate the conversion of PYY(1-36) to PYY(3-36) and, as a result, possibly increase meal size and weight gain. Clearly, this would be an undesirable effect of DP IV inhibitor therapy, especially in subjects with type 2 diabetes, who are typically overweight. We examined the effects of PYY(1-36) and PYY(3-36) on food intake of normal and DP IV-deficient rats. The effects of a DP IV inhibitor, Pro-boroPro, on the generation of PYY(3-36) from PYY (1-36) in vitro and in vivo were also studied and we investigated the ability of exogenous PYY(1-36) to regulate food intake in the presence of Pro-boroPro. Finally, feeding pattern, food intake and periprandial PYY levels were studied in normal and DP IV-deficient rats after a 24-h fast. Our results indicate that DP IV is a key enzyme responsible for the generation of PYY(3-36), the anorectic form of PYY, yet short-term inhibition of DP IV using ProboroPro does not affect the satiety effects of exogenous PYY $(1-36)$ at the doses tested.

\section{Materials and methods}

\section{Animals}

Fischer 344 rats (normal rats; 200-225 g) were purchased from Charles River Laboratories (Saint-Constant, QC, Canada). DP IV-negative Fischer 344 rats (DP IV-deficient rats; 200-225 g) were obtained from a breeding colony maintained in our animal facility. DP IV-deficient rats lack DP IV [33] and have been previously characterised [34]. In all studies, age- and weight-matched male rats were individually housed in grid cages in a 12-h light (08.00$20.00 \mathrm{~h}) / 12-\mathrm{h}$ darkness $(20.00-08.00 \mathrm{~h})$ photoperiod at $23 \pm 1^{\circ} \mathrm{C}$ and controlled humidity in our animal care facility. Unless otherwise stated, animals had free access to tap water and rat chow (Purina Mills, St Louis, MO, USA). Rat chow was provided on the floor of grid cages and this allowed us to collect residual food particles on a tray kept beneath cages, enabling precise monitoring of food intake. Research protocols used in this study were approved by the Animal Care Committee of our university in compliance with the Canadian Council for Animal Care guidelines.

Materials

Rat PYY(1-36) (Catalogue no. 059-03), rat PYY(3-36) (Catalogue no. 059-04) and human PYY(1-36) (Catalogue no. 059-01) were purchased from Phoenix Pharmaceuticals (Belmont, CA, USA). Human PYY(1-36) and DP IV used for the matrix-assisted laser desorption/ionisation (MALDI)-time-of-flight (TOF) mass spectrometry studies and Pro-boroPro $\left(\mathrm{C}_{9} \mathrm{H}_{17} \mathrm{BN}_{2} \mathrm{O}_{3} ; 98.6 \%\right.$ purity $)$ were synthesised at Probiodrug (Halle, Germany). All peptides were HPLC purified to $\geq 95 \%$ purity. Peptides were lot-matched when multiple vials were required in a study and were freshly prepared in $0.9 \%$ saline for each study. One-day (Model 2001D) and 14-day (Model 2ML2) Alzet osmotic mini-pumps were purchased from Durect Corporation (Cupertino, CA, USA). A total PYY ELISA kit (Catalogue no. DSL-10-33600), which detects both PYY(1-36) and PYY(3-36), was purchased from DS Labs (Webster, TX, USA) and the human PYY(3-36)-specific RIA kit was purchased from Linco Research Inc. (St Charles, MO, USA). Chocolate-flavoured Ensure (Abbott Laboratories, Saint-Laurent, QC, Canada) was purchased from a local pharmacy. 
Study design

Circulating levels of total PYY during continuous infusion of $P Y Y(3-36)$ in normal rats Normal rats were implanted with osmotic mini-pumps (1 day) containing saline $(n=6)$ or $100 \mu \mathrm{g} \cdot \mathrm{kg}$ body weight ${ }^{-1}$. $^{-1}{ }^{-1}$ PYY(3-36) $(n=6)$. Blood samples were collected at $0,3,9,18$ and $24 \mathrm{~h}$ after implantation. Plasma was separated and stored at $-20^{\circ} \mathrm{C}$ until the PYY ELISA was conducted.

Effects of continuous infusion of PYY(3-36) for 6 days on food intake and weight gain of non-acclimatised normal rats Osmotic mini-pumps (14-day) infusing $100 \mu \mathrm{g} \cdot \mathrm{kg}$ body weight ${ }^{-1} \cdot$ day $^{-1}$ PYY(3-36) $(n=6)$ or saline $(n=6)$ were implanted in normal rats and daily food intake and body weight were measured for 6 days after implantation. Since only a transient effect of PYY(3-36) on food intake was seen, we terminated the experiment at 6 days after implantation. To identify the effects of surgical stress and implantation of osmotic mini-pumps on food intake, we used non-acclimatised rats for this study.

In vitro effects of DP IV inhibitor on the generation of PYY (3-36) from PYY(1-36) To obtain the mass spectra of PYY (1-36) degradation by DP IV, human PYY(1-36) $(62.5 \mu \mathrm{mol} / \mathrm{l})$ was incubated at $37^{\circ} \mathrm{C}$ with $0.1 \mathrm{~mol} / 1$ Tris buffer ( $\mathrm{pH}$ 7.6) containing DP IV plus water or DP IV plus Pro-boroPro in a 2:2:1 ratio. Samples $(10 \mu \mathrm{l})$ from the incubation mixture were collected at $180 \mathrm{~min}$ and mixed with equal volumes of $0.1 \%$ trifluoroacetic acid solution. Five microlitres of the elution solution were mixed with $5 \mu 1$ MALDI matrix solution. Next, $1 \mu \mathrm{l}$ of this mixture was transferred to a probe tip for sample crystallisation. MALDI-TOF mass spectrometry was carried out using a mass spectrometer (Voyager-DE Pro; Applied Biosystems, Darmstadt, Germany) in a positive reflector mode. The spectrometer was externally calibrated using the calmix 2 standard solution of Applied Biosystems. Spectra of 300 single shots per sample were accumulated.

Effects of Pro-boroPro on plasma DP IV activity in normal rats Pro-boroPro $(1,000 \mu \mathrm{g} / \mathrm{rat})$ dissolved in saline $(n=4)$, or saline alone $(n=4)$, was injected s.c. at $0 \mathrm{~h}$ into normal rats. The same dose of Pro-boroPro was injected again after $12 \mathrm{~h}$. Blood was collected at $0,3,12$ and $24 \mathrm{~h}$ from the tail vein and plasma separated by centrifugation at $10,000 \mathrm{~g}$ for 9 min at $4{ }^{\circ} \mathrm{C}$ and kept at $-20^{\circ} \mathrm{C}$ until plasma DP IV activity was measured using a previously described protocol [2].

Role of DP IV in generating PYY(3-36) from PYY(1-36) in vivo Osmotic mini-pumps infusing $100 \mu \mathrm{g} \cdot \mathrm{kg}$ body weight $^{-1} \cdot$ day $^{-1}$ human PYY(1-36) were implanted into normal rats $(n=6)$, DP IV-deficient rats $(n=6)$ and normal rats injected with Pro-boroPro at the time of implantation $(0 \mathrm{~h})$. Blood samples were collected at 0 and $3 \mathrm{~h}$ after implantation into microfuge tubes containing DP IV inhibitor and aprotinin. Plasma was separated and stored at $-20^{\circ} \mathrm{C}$ until an RIA specific for human PYY(3-36) was conducted. Background human PYY(3-36) immunoreactivity was subtracted from all values.

Effects of PYY(1-36) and PYY(3-36) on food intake of normal and DP IV-deficient rats Normal rats and DP IVdeficient rats were acclimatised for 7 days prior to the implantation of osmotic mini-pumps. Animals were kept in grid cages for 4 days from the day of arrival. On day 5 (day 1 of acclimatisation), animals were transferred to the procedure room on a cart, anaesthetised by inhalation of 3\% isoflurane using oxygen as gaseous carrier, shaved in the area where the incision was to be made, and weighed. Once removed from the anaesthetic machine, animals quickly recovered from anaesthesia and were then returned to the animal facility. Food intake was measured by deducting the quantity of food recovered after 24-h feeding from the initial amount of food given. Acclimatisation (except shaving) was repeated daily for the next 6 days. Rats were then implanted with osmotic mini-pumps (1 day) containing saline $(n=6), \operatorname{PYY}(1-36)\left(100 \mu \mathrm{g} \cdot \mathrm{kg}\right.$ body weight ${ }^{-1}$. day $\left.^{-1} ; n=6\right)$ or PYY (3-36) (100 $\mu \mathrm{g} \cdot \mathrm{kg}$ body weight ${ }^{-1}$. day $\left.^{-1} ; n=6\right)$. The cumulative food intake for $24 \mathrm{~h}$ after implantation was measured.

Effects of Pro-boroPro on satiety actions of PYY in normal rats Normal rats were acclimatised as described previously and implanted with osmotic mini-pumps (1 day) infusing saline $(n=6)$, PYY $(1-36)\left(100 \mu \mathrm{g} \cdot \mathrm{kg}\right.$ body weight ${ }^{-1} \cdot \mathrm{day}^{-1}$; $n=6)$ or PYY (3-36) (100 $\mu \mathrm{g} \cdot \mathrm{kg}$ body weight $\left.{ }^{-1} \cdot \mathrm{day}^{-1} ; n=6\right)$. A fourth group received pumps infusing PYY(1-36) (100 $\mu \mathrm{g} \cdot \mathrm{kg}$ body weight $\left.{ }^{-1} \cdot \mathrm{day}^{-1} ; n=6\right)$ and two s.c. injections of 1,000 $\mu \mathrm{g}$ Pro-boroPro at 12-h intervals, while the fifth group $(n=6)$ received two s.c. injections of $1,000 \mu \mathrm{g}$ Pro-boroPro at 12 -h intervals. In addition to the usual acclimatisation procedures, rats used in this study were acclimatised to i.p. injections of saline twice daily for 3 days prior to the experiment day.

Periprandial PYY levels in DP IV-positive and-deficient rats Normal $(n=6)$ and DP IV-deficient $(n=6)$ rats were fasted for $24 \mathrm{~h}$ from $07.00 \mathrm{~h}$ and gavaged with $4 \mathrm{ml}$ Ensure (4.25 calories). Blood samples were collected at 0, 30, 60, 90,150 and $210 \mathrm{~min}$ and plasma was immediately separated and kept at $-20^{\circ} \mathrm{C}$ until total PYY were measured by ELISA following the manufacturer's instructions.

Food intake and weight gain of normal and DP IV-deficient rats re-fed after a 24- $h$ fast Normal $(n=6)$ and DP IV- 


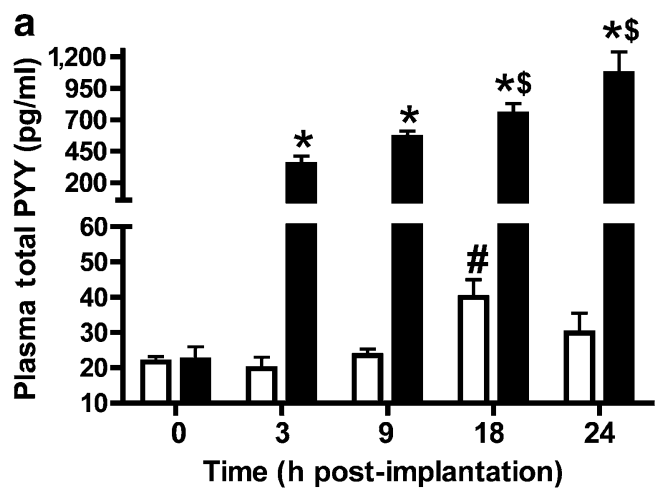

b

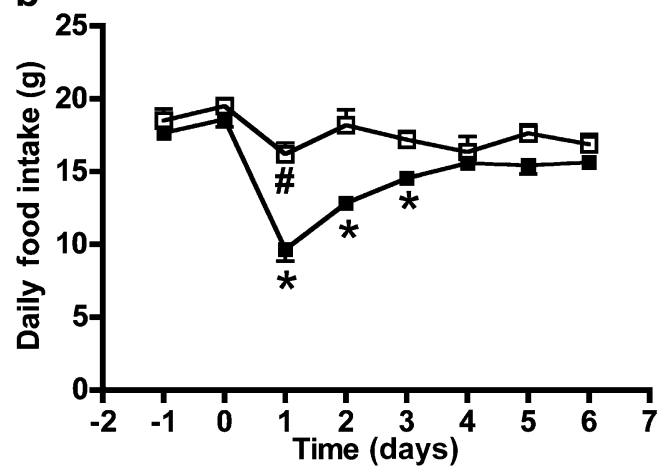

C

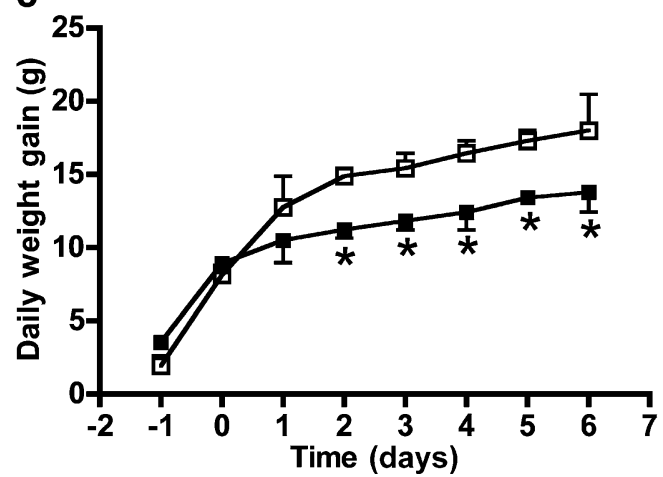

Fig. 1 a The 24-h profile of total PYY in the circulation in normal rats continuously infused with saline (white bars) or $100 \mu \mathrm{g} \cdot \mathrm{kg}$ body weight $^{-1} \cdot$ day $^{-1}$ PYY(3-36) (black bars). Data are means \pm SEM. $n=6$ rats/group. ${ }^{*} p<0.05$, saline vs treatment groups; $\$ p<0.05$, PYY-treated group at $18 \mathrm{~h}(06.00 \mathrm{~h})$ and $24 \mathrm{~h}(12.00 \mathrm{~h})$ vs the PYY-treated group at $0 \mathrm{~h}(12.00 \mathrm{~h})$ and $3 \mathrm{~h}(15.00 \mathrm{~h}) ;{ }^{*} p<0.05$, saline-treated group at $18 \mathrm{~h}$ $(06.00 \mathrm{~h})$ vs the saline-treated group at 0,3 and $9 \mathrm{~h}$. b, c Effects of s.c. infusion of $100 \mu \mathrm{g} \cdot \mathrm{kg}$ body weight ${ }^{-1} \cdot \mathrm{day}^{-1} \operatorname{PYY}(3-36)$ (solid squares) or saline (open squares) for 6 days on daily food intake (b) and body weight gain (c) of normal rats. Data are means \pm SEM. $n=6$ rats/group. ${ }^{*} p<0.05$, saline vs treatment groups; ${ }^{*} p<0.05$, saline day 0 vs saline day 1

deficient $(n=6)$ rats were fasted for $24 \mathrm{~h}$ from 08.00 to $20.00 \mathrm{~h}$, re-fed with pre-weighed quantities of food and hourly food intake was monitored for $12 \mathrm{~h}$. Cumulative food intake for 12 to $24 \mathrm{~h}$ and body weight gain at $24 \mathrm{~h}$ after re-feeding were also recorded.
Statistical analyses

All data are presented as means \pm SEM. Data were analysed using either a paired $t$-test or an ANOVA followed by a Student-Newman-Keuls test or Dunnet's multiple comparison test. $p<0.05$ was considered statistically significant. Graphing and statistical analyses were conducted using GraphPad Prism Version 4 (GraphPad Software Inc., San Diego, CA, USA).

\section{Results}

Circulating levels of total PYY during continuous infusion of PYY(3-36) in normal rats

Continuous infusion of PYY(3-36) caused a significant increase in total PYY levels compared with saline-treated controls at 3, 9, 18 and $24 \mathrm{~h}$ after implantation (Fig. 1a). Total PYY levels in PYY(3-36)-infused animals were approximately 20-, 18-, 13- and 27-fold higher than in saline-treated controls at 3,9, 18 and $24 \mathrm{~h}$ after implantation, respectively. Total PYY levels in PYY-treated animals at 18 and $24 \mathrm{~h}$ after implantation were significantly higher than PYY levels at $3 \mathrm{~h}$. In saline-treated controls, total PYY levels were significantly higher ( 2-fold increase) at $18 \mathrm{~h}$ $(06.00 \mathrm{~h})$ than $0 \mathrm{~h}$ and all sampling points after implantation, indicating a postprandial surge in endogenous PYY in normal rats.

Effects of continuous infusion of PYY(3-36) for 6 days on food intake and body weight of non-acclimatised normal rats

Continuous infusion of PYY(3-36) for 6 days caused a transient reduction in food intake (Fig. 1b) and a prolonged reduction in weight gain (Fig. 1c) compared with the salinetreated control animals. PYY-treated animals had $\sim 20-30 \%$ reduction in food intake on days 1 to 3 after implantation of osmotic pumps, while $\sim 20 \%$ reduction in weight gain was observed throughout the study period. Food intake of saline-treated rats on day 1 after implantation was significantly lower than food intake on the day immediately before surgery and implantation of pumps (Fig. 1b), indicating stress-induced anorexia in these rats.

In vitro effects of DP IV inhibitor on the generation of PYY (3-36) from PYY(1-36)

Incubation of PYY(1-36) with DP IV resulted in the cleavage of PYY(1-36) to PYY(3-36) at $180 \mathrm{~min}$ after incubation (Fig. 2a). Co-incubation with the DP IV inhibitor, Pro-boroPro, abolished the formation of PYY(3- 

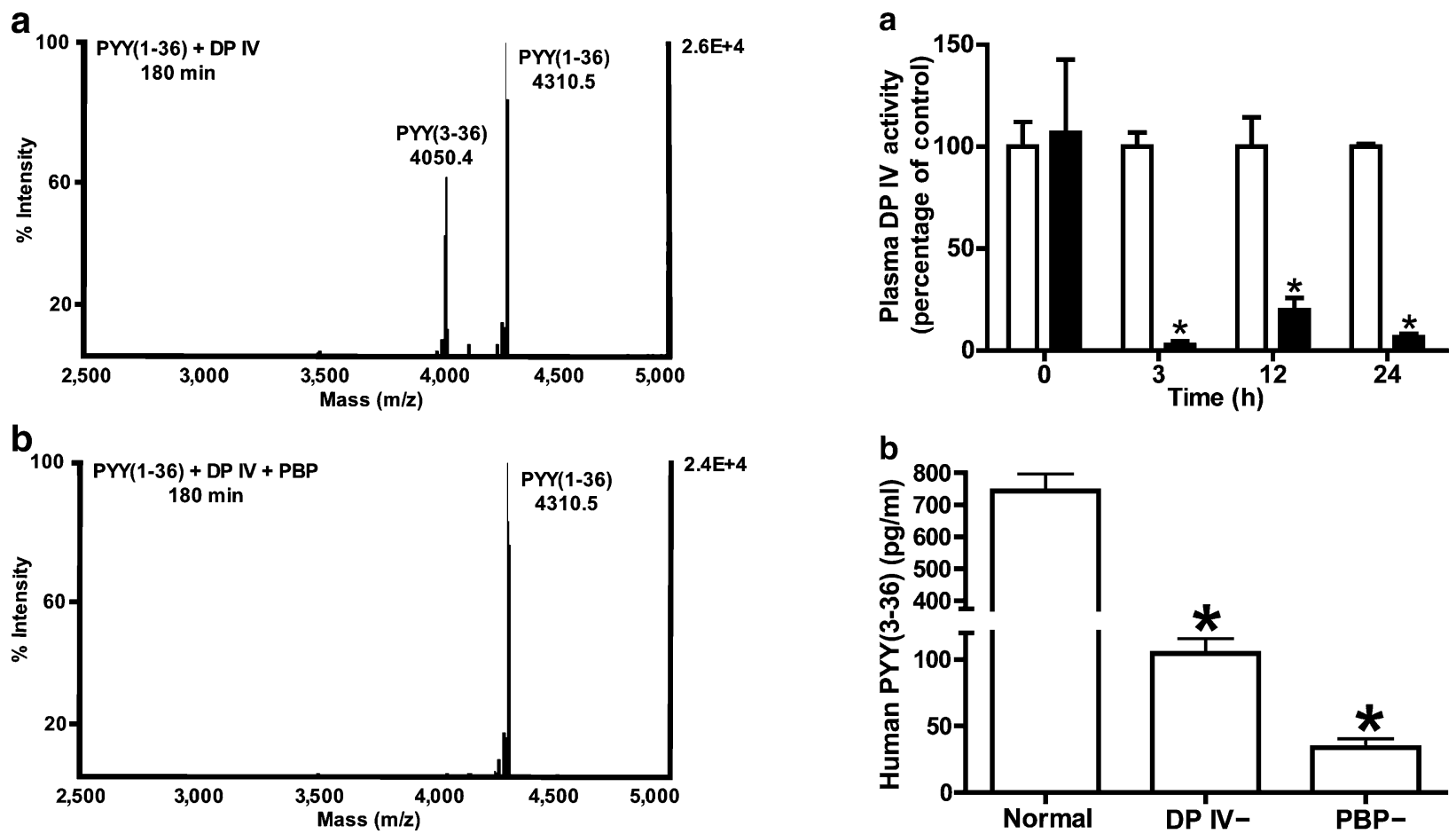

Fig. 2 MALDI-TOF mass spectrometry analysis of DP IV-catalysed hydrolysis of PYY(1-36) in the absence (a) or presence (b) of the inhibitor Pro-boroPro $(P B P)$. Synthetic human PYY $(1-36)\left([\mathrm{M}+\mathrm{H}]^{+}=\right.$ 4,310.4) was incubated with purified human recombinant DP IV. Samples were removed from the incubation mixture and analyte immediately crystallised with sinapinic acid as matrix and analysed by mass spectrometry. The presence of Pro-boroPro completely inhibited DP IV-induced conversion of PYY(1-36) to PYY(3-36) over a 3-h incubation period

36) (Fig. 2b). These results indicate that Pro-boroPro is a potent DP IV inhibitor and it attenuates the DP IV-mediated conversion of PYY(1-36) to PYY(3-36).

\section{Effects of Pro-boroPro on plasma DP IV activity} in normal rats

Subcutaneous injections of Pro-boroPro caused $\sim 97 \%$ reduction in plasma DP IV activity at $3 \mathrm{~h}$ after injection, $\sim 80 \%$ reduction at $12 \mathrm{~h}$ after injection and $\sim 93 \%$ reduction at $24 \mathrm{~h}$ after injection (Fig. 3a). There was no difference in plasma DP IV activity between study groups at $0 \mathrm{~h}$ (Fig. 3a). Pro-boroPro, at the dose tested, did not cause any visible symptoms of toxicity in the treatment group during the 24-h study period. Treated rats were monitored for 1 week following the study period with no findings of toxicity.

Role of DP IV in generating PYY(3-36)

from PYY(1-36) in vivo

Pharmacological levels of human PYY(3-36) were found in the circulation at $3 \mathrm{~h}$ after infusion of human PYY $(1-36)$

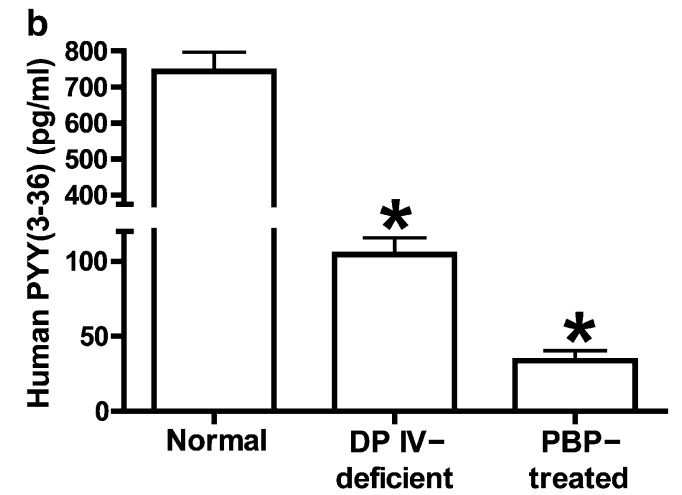

Fig. 3 a Effects of two s.c. injections of 1,000 $\mu \mathrm{g}$ Pro-boroPro at 12-h intervals on plasma DP IV activity in normal rats. White bars, saline; black bars, Pro-boroPro. Data are means \pm SEM. $n=4$ rats/ group. ${ }^{*} p<0.001$, saline vs Pro-boroPro. b Circulating levels of human PYY(3-36) after the administration of human PYY(1-36) to normal and DP IV-deficient rats, and to rats treated with the DP IV inhibitor, Pro-boroPro, at $3 \mathrm{~h}$ after implantation of human PYY(1-36)-infusing osmotic mini-pumps. Data are means \pm SEM. $n=6$ rats/group. Overall $p$ value for one-way ANOVA, $p<0.001$. ${ }^{*} p<0.01$ compared with normal rats

into normal rats (Fig. 3b). Detectable quantities of PYY(336) were also found in DP IV-deficient rats and in ProboroPro-treated rats (Fig. 3b). Human PYY(3-36) levels in Pro-boroPro-treated rats and DP IV-deficient rats were significantly lower than the human PYY(3-36) levels in normal rats, indicating the attenuated conversion of PYY (1-36) to PYY(3-36) due to the absence/inhibition of DP IV enzyme.

Effects of PYY(1-36) and PYY(3-36) on food intake of normal and DP IV-deficient rats

In these acclimatised rats, saline infusion did not significantly alter food intake relative to the day prior to pump implantation $(17.95 \pm 0.85$ vs $17.03 \pm 0.43 \mathrm{~g} /$ day, respectively). At a dose of $100 \mu \mathrm{g} \cdot \mathrm{kg}$ body weight ${ }^{-1} \cdot$ day $^{-1}$, both PYY (1-36) and PYY(3-36) administration significantly reduced $(\sim 20 \%)$ 24-h cumulative food intake in normal rats compared with saline-treated controls (Fig. 4a). In contrast, PYY(3-36), but not PYY(1-36), reduced food intake in DP IV-deficient rats compared with saline controls 

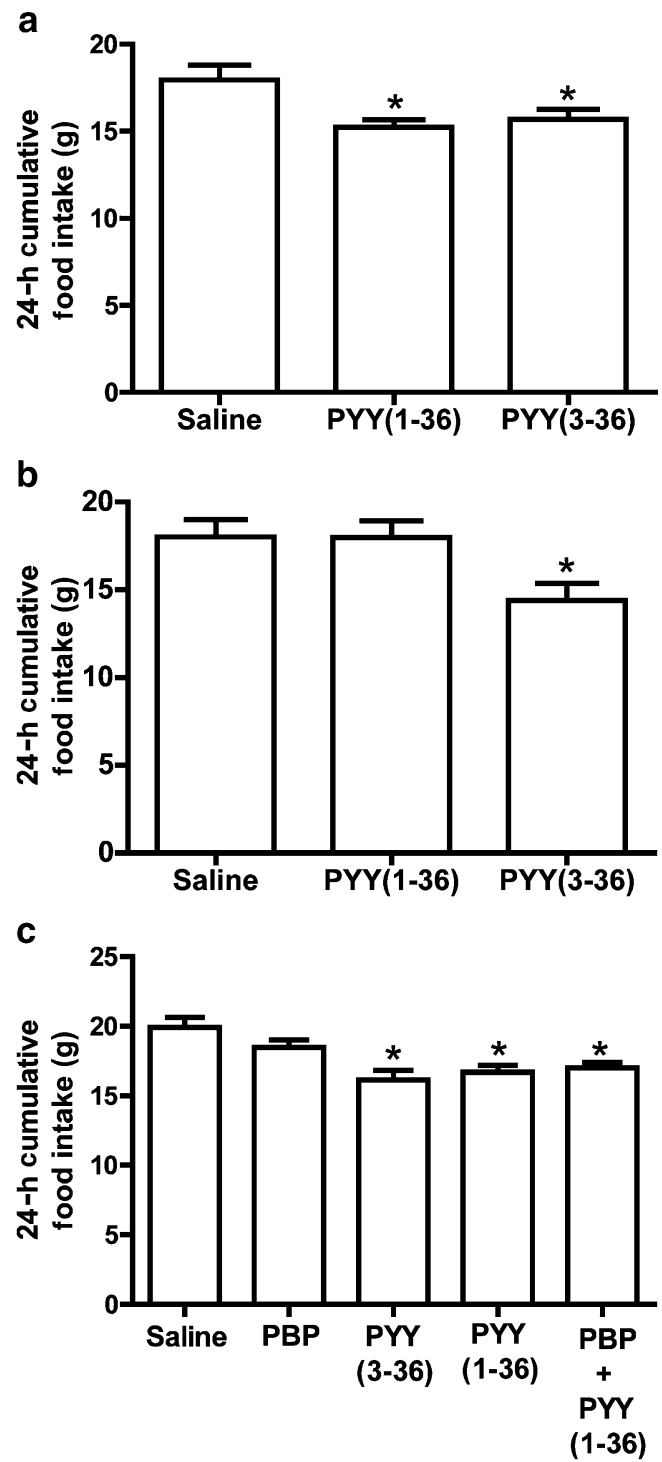

Fig. 4 Effects of s.c. infusion of $100 \mu \mathrm{g} / \mathrm{kg}$ body weight PYY(1-36) or PYY(3-36) for 1 day on food intake of normal rats (a) and DP IVdeficient rats (b). Data are means \pm SEM. $n=6$ rats/group. ${ }^{*} p<0.05$, saline vs treatment groups. c Effects of co-administration of $1,000 \mu \mathrm{g}$ DP IV inhibitor (Pro-boroPro; PBP) and $100 \mu \mathrm{g} / \mathrm{kg}$ body weight PYY (1-36) on food intake of normal rats. In the PBP group and PBP+PYY (1-36)-treated group, two s.c. injections of PBP were given to normal rats in addition to PYY $(1-36)$ infusion via osmotic pumps. Data are means \pm SEM. $n=6$ rats/group. Overall $p$ value for one-way ANOVA, $p<0.007 .{ }^{*} p<0.01$, saline vs treatment groups

(Fig. 4b). At a dose of $1,000 \mu \mathrm{g} / \mathrm{kg}$ body weight, PYY(336) administration reduced 24-h cumulative food intake by $\sim 40 \%$ in normal rats (not shown).

Effects of Pro-boroPro on satiety actions of PYY in normal rats

Both PYY(3-36) and PYY(1-36) caused a significant reduction $(\sim 20 \%)$ in 24 -h cumulative food intake following implantation compared with saline-treated controls
(Fig. 4c). Co-administration of PYY(1-36) and ProboroPro also caused a significant reduction in food intake, indicating that at the doses tested Pro-boroPro has no inhibitory actions on PYY's satiety effects (Fig. 4c). A nonsignificant reduction in food intake was also observed in the Pro-boroPro-treated group (Fig. 4c).

Periprandial PYY levels in normal and DP IV-negative rats

DP IV-deficient rats exhibited significantly higher plasma total PYY levels than normal rats at $0 \mathrm{~h}$ (Fig. 5a). In normal rats, plasma total PYY levels significantly increased $30 \mathrm{~min}$ after re-feeding compared with basal levels (Fig. 5a) and remained significantly elevated over basal levels at 60 and 90 min after re-feeding (Fig. 5a). No periprandial changes in plasma PYY levels were found in DP IV-deficient rats.

Food intake and weight gain of normal and DP IV-negative rats re-fed after a 24-h fast

Food intake of DP IV-deficient rats at $2 \mathrm{~h}$ after re-feeding was significantly higher than normal rats (Fig. 5b). Cumulative food intake during the light phase (Fig. 5c) and dark phase (Fig. 5c) was also significantly higher in DP IVdeficient rats compared with normal rats. Weight gain during 24-h after re-feeding was significantly higher in DP IV-deficient rats compared with normal rats (Fig. 5d).

\section{Discussion}

DP IV inhibitors are being explored as agents to extend the biological activity of insulinotropic hormones; at the same time, they may attenuate activities of other hormones. Although the effect of DP IV inhibition on GIP and GLP-1 and its therapeutic potential in treating diabetes are well studied, few studies have examined the effects of DP IV inhibition on other peptidyl substrates of DP IV in vivo [35]. The studies discussed here analyse the effects of shortterm DP IV inhibition on the satiety effects of PYY, a substrate of DP IV, as well as PYY responses and food intake in DP IV-deficient rats.

PYY is under intense study due to its ability to reduce food intake and thereby promote weight loss [18, 24]. However, several contradictory reports exist regarding the satiety effects of PYY(3-36) in rodents [22]. In our hands, continuous infusion of PYY(3-36) for 6 days caused a significant but transient reduction in food intake accompanied by a prolonged reduction in weight gain. We also found a significant reduction in the food intake of salinetreated animals on day 1 after implantation in our 6-day study. However, this effect was gone by day 2 after implantation and was absent in acclimatised rats, indicating 
Fig. 5 a Periprandial plasma total PYY levels in normal and DP IV-deficient rats. Arrow indicates time of meal gavage. Data are means \pm SEM. $n=6$ rats group. ${ }^{\#} p<0.05$, PYY levels at $0 \mathrm{~h}$ in DP IV-deficient rats vs normal rats; ${ }^{*} p<0.05$, PYY levels at a time-point compared with the basal PYY levels at $0 \mathrm{~h}$. b Hourly food intake of normal and DP IV-deficient rats for $12 \mathrm{~h}$ following a 24-h fast; c cumulative food intake of normal and DP IV-deficient rats during the dark phase and light phase; and d cumulative weight gain after 24-h re-feeding. Black squares/ bars, DP IV-deficient rats; open circles/bars, normal rats. Data are means \pm SEM. $n=6$ rats/ group. ${ }^{*} p<0.05$, DP IV-deficient rats vs normal rats
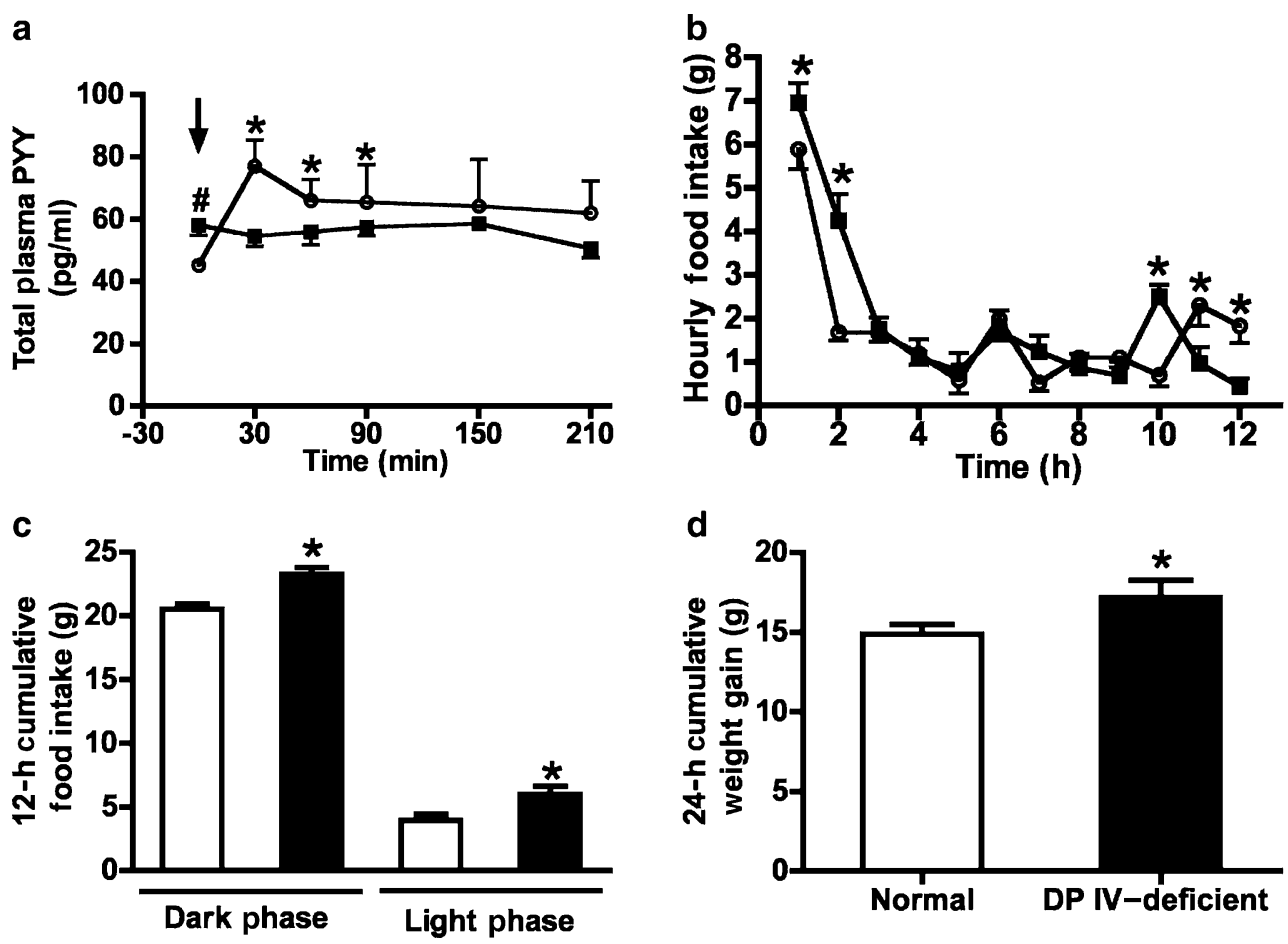

that it results from stress associated with implantation of the osmotic pump. Pumps delivering pharmacological levels of PYY(3-36) caused significantly greater and more prolonged reductions in food intake than in the saline-treated animals. This indicates that PYY(3-36) caused an anorectic effect greater than surgical stress-induced anorexia in our animals, in agreement with previously reported studies [23, 24].

Interestingly, while both PYY(1-36) and PYY(3-36) inhibited food intake of normal rats, PYY(1-36) had no effect on food intake of DP IV-deficient rats, suggesting that DP IV is required for generating the anorexigen, PYY (3-36). Our in vitro studies with purified DP IV confirm that this enzyme can indeed convert PYY(1-36) to PYY(336) in vitro and that Pro-boroPro, a DP IV inhibitor [36, 37], completely inhibited this conversion. Thus, inhibition of DP IV in vivo may also reduce the production of endogenous PYY(3-36).

Despite the fact that PYY(1-36) had no satiety effects in DP IV-deficient rats, suppression of DP IV activity in normal rats with Pro-boroPro did not attenuate the satiety actions of exogenous PYY(1-36). This result could be due to several reasons. Firstly, although a potent DP IV inhibitor was used, complete inhibition of DP IV in plasma was not achieved during the 24-h study period. This is indicated in our studies where at $3 \mathrm{~h}$ after administration of Pro-boroPro and human PYY(1-36), low but significant amounts of immunoreactive human PYY(3-36) were still produced despite the fact that approximately $97 \%$ reduction in plasma DP IV activity was achieved. Moreover, DP IV is a ubiquitous enzyme, lining the vasculature in abundance, including the microvasculature in close proximity to the PYY-producing L-cells [38]. Aside from in plasma, significant DP IV activity could remain on the vasculature such that exogenous PYY (1-36) could have been cleaved to PYY(3-36). Secondly, it is possible that enzymes other than DP IV could also generate PYY(3-36) from PYY(136), as PYY(3-36) is formed even in the DP IV-deficient rats. Thirdly, inhibition of DP IV would be anticipated to alter the intact levels of other regulatory peptides that could alter food intake. Indeed administration of the inhibitor alone tended to reduce food intake in our studies. One candidate hormone is GLP-1, as both central [39] and peripheral administration of GLP-1 inhibits food intake in rodents [40, 41]. GLP-1 is a substrate of DP IV and circulating levels of the active form of GLP-1 increase following the administration of DP IV inhibitors in rats [42]. Although we did not measure the circulating levels of GLP-1 levels following DP IV inhibition, such an increase in bioactive GLP-1 levels due to DP IV inhibition might also have contributed to the reduction in food intake of rats co-administered PYY(1-36) and Pro-boroPro.

Compensation can occur due to long-term DP IV deficiency, including altered levels of regulatory peptides [34] and sensitivity to their actions [35]. Cumulative food intake during the dark phase and light phase and 24-h cumulative weight gain after a 24-h fast were higher in DP IV-deficient rats than in control animals. Thus there is mild hyperphagia and increased weight gain in DP IVdeficient rats when re-fed after a 24-h fast, possibly a result of the reduced levels of the short-term satiety signal, PYY 
(3-36). We also found that, in contrast to normal rats, circulating total PYY levels were elevated under basal conditions and did not increase postprandially in DP IVdeficient rats. The absent response in DP IV-deficient rats could be an adaptive/protective response to the extended half-life of PYY(1-36) in these animals. Notably, it has been previously reported that DP IV-deficient rats have lower secretion of GLP-1 and GIP compared with normal rats following an OGTT [34].

Using PYY as an example, the present study demonstrates that DP IV plays a major role in the conversion of PYY(1-36) to PYY(3-36) and DP IV inhibition attenuates this conversion. However, our results from DP IV-deficient rats suggest possible roles of other dipeptidyl peptidases in regulating the generation of PYY(1-36) to PYY(3-36). Although co-administration of PYY with a DP IV inhibitor did not change the satiety effects of PYY(1-36), the anorectic effects of PYY(1-36) were attenuated in DP IVdeficient rats and they had higher food intake and weight gain when re-fed after a 24-h fast. It remains to be determined whether acute/chronic treatment with DP IV inhibitors influences the endogenous production of PYY(336). Since the type of inhibitor, specificity of the inhibitor used, doses administered and the mode of administration are important factors affecting the efficacy of DP IV inhibitors, further studies using various animal models, inhibitors and peptide substrates are necessary to elucidate the effects of DP IV inhibitors on physiological processes regulated by DP IV substrates. Long-term administration of DP IV inhibitor in rats (100 days) [42] or humans (52 weeks) [43] did not alter cumulative food intake or body weight. In contrast, a 6-week therapy using GLP-1 [44] or a 28-day therapy using a GLP-1 mimetic, exenatide [45], caused significant reductions in body weight of humans. Although DP IV inhibition increases intact bioactive GLP-1 levels, and thus might be anticipated to reduce food intake, it also attenuates the conversion of endogenous PYY(1-36) to PYY(3-36), as well as potentially altering the levels of several other regulatory peptides that control food intake. Thus, it appears that the net effect of short-term DP IV inhibition on satiety is neutral.

Acknowledgements Sincere thanks are due to M. Speck and T. D. Webber for excellent technical assistance. T. J. Kieffer is a Michael Smith Foundation for Health Research (MSFHR) Scholar and the recipient of a Career Development Award from the Juvenile Diabetes Research Foundation. S. Unniappan is a recipient of postdoctoral fellowships from the Canadian Institutes of Health Research (CIHR) and the MSFHR. T. J. Kieffer and C. H. S. McIntosh acknowledge grant support from CIHR. H.-U. Demuth acknowledges grant support from the German state of Saxony-Anhalt.

Duality of Interest The authors declare that they have no duality of interest relating to this manuscript.

\section{References}

1. Lambeir AM, Durinx C, Scharpe S, De Meester I (2003) Dipeptidyl-peptidase IV from bench to bedside: an update on structural properties, functions, and clinical aspects of the enzyme DPP IV. Crit Rev Clin Lab Sci 40:209-294

2. Kieffer TJ, McIntosh CH, Pederson RA (1995) Degradation of glucose-dependent insulinotropic polypeptide and truncated glucagon-like peptide 1 in vitro and in vivo by dipeptidyl peptidase IV. Endocrinology 136:3585-3596

3. McIntosh CH, Demuth HU, Pospisilik JA, Pederson R (2003) Dipeptidyl peptidase IV inhibitors: how do they work as new antidiabetic agents? Regul Pept 128:159-165

4. Drucker DJ (2003) Enhancing incretin action for the treatment of type 2 diabetes. Diabetes Care 26:2929-2940

5. Lenhard JM, Gottschalk WK (2002) Preclinical developments in type 2 diabetes. Adv Drug Deliv Rev 54:1199-1212

6. Scharpe S, De Meester I (2001) Peptide truncation by dipeptidyl peptidase IV: a new pathway for drug discovery? Verh K Acad Geneeskd Belg 63:5-33

7. Adrian TE, Ferri GL, Bacarese-Hamilton AJ, Fuessl HS, Polak JM, Bloom SR (1985) Human distribution and release of a putative new gut hormone, peptide YY. Gastroenterology 89:1070-1077

8. Grandt S, Teyssen S, Schimiczek M et al (1992) Novel generation of hormone receptor specificity by amino terminal processing of peptide YY. Biochem Biophys Res Commun 186:1299-1306

9. Mederois MD, Turner AJ (1994) Processing and metabolism of peptide-YY: pivotal roles of dipeptidylpeptidase-IV, aminopeptidase-P, and endopeptidase-24.11. Endocrinology 134:2088-2094

10. Mentlein R, Dahms P, Grandt D, Kruger R (1993) Proteolytic processing of neuropeptide $\mathrm{Y}$ and peptide $\mathrm{YY}$ by dipeptidyl peptidase IV. Regul Pept 49:133-144

11. Mentlein R (1999) Dipeptidyl-peptidase IV (CD26) - role in the inactivation of regulatory peptides. Regul Pept 85:9-24

12. Playford RJ, Mehta S, Upton P et al (1995) Effect of peptide YY on human renal function. Am J Physiol 268:F754-F759

13. Playford RJ, Benito-Orfila MA, Nihoyannopoulos P et al (1992) Effects of peptide YY on the human cardiovascular system: reversal of responses to vasoactive intestinal peptide. Am J Physiol 263:E740-E747

14. Ferrier L, Segain JP, Pacaud P et al (2000) Pathways and receptors involved in peptide $\mathrm{YY}$ induced contraction of rat proximal colonic muscle in vitro. Gut 46:370-375

15. Yang H (2002) Central and peripheral regulation of gastric acid secretion by peptide YY. Peptides 23:349-358

16. Naruse S, Kitagawa M, Ishiguro H, Hayakawa T (2002) Feedback regulation of pancreatic secretion by peptide YY. Peptides 23:359-365

17. Strader AD, Woods SC (2005) Gastrointestinal hormones and food intake. Gastroenterology 128:175-191

18. Batterham RL, Cowley MA, Small CJ et al (2002) Gut hormone PYY(3-36) physiologically inhibits food intake. Nature 418:650-654

19. Challis BG, Coll AP, Yeo GS et al (2004) Mice lacking proopiomelanocortin are sensitive to high-fat feeding but respond normally to the acute anorectic effects of peptide-YY(3-36). Proc Natl Acad Sci USA 101:4695-4700

20. Halatchev IG, Cone RD (2005) Peripheral administration of PYY3-36 produces conditioned taste aversion in mice. Cell Metabolism 1:159-168

21. Abbott CR, Monteiro M, Small CJ et al (2005) The inhibitory effects of peripheral administration of peptide $\mathrm{YY}(3-36)$ and glucagon-like peptide- 1 on food intake are attenuated by ablation 
of the vagal-brainstem-hypothalamic pathway. Brain Res 1044:127-131

22. Tschop M, Castaneda TR, Joost HG et al (2004) Does gut hormone PYY3-36 decrease food intake in rodents? Nature 430:165-166

23. Pittner RA, Moore CX, Bhavsar SP et al (2004) Effects of PYY (3-36) in rodent models of diabetes and obesity. Int J Obes Relat Metab Disord 28:963-971

24. Chelikani PK, Haver AC, Reidelberger RD (2005) Intravenous infusion of peptide YY(3-36) potently inhibits food intake in rats. Endocrinology 146:879-888

25. Moran TH, Smedh U, Kinzig KP, Scott KA, Knipp S, Ladenheim EE (2005) Peptide YY(3-36) inhibits gastric emptying and produces acute reductions in food intake in rhesus monkeys. Am J Physiol Regul Integr Comp Physiol 288:R384-R388

26. Batterham RL, Cohen MA, Ellis SM et al (2003) Inhibition of food intake in obese subjects by peptide YY3-36. N Engl J Med 349:941-948

27. Jobst EE, Enriori PJ, Cowley MA (2004) The electrophysiology of feeding circuits. Trends Endocrinol Metab 15:488-499

28. Koda S, Date Y, Murakami N (2005) The role of the vagal nerve in peripheral PYY3-36-induced feeding reduction in rats. Endocrinology 146:2369-2375

29. Chelikani PK, Haver AC, Reidelberger RD (2004) Comparison of the inhibitory effects of PYY(3-36) and PYY(1-36) on gastric emptying in rats. Am J Physiol Regul Integr Comp Physiol 287: R1064-R1070

30. Morley JE, Levine AS, Grace M, Kneip J (1985) Peptide YY (PYY), a potent orexigenic agent. Brain Res 341:200-203

31. Stanley BG, Daniel DR, Chin AS, Leibowitz SF (1985) Paraventricular nucleus injections of peptide YY and neuropeptide Y preferentially enhance carbohydrate ingestion. Peptides 6:1205-1211

32. Lin S, Boey D, Herzog H (2004) NPY and Y receptors: lessons from transgenic and knockout models. Neuropeptides 38:189-200

33. Watanabe Y, Kojima T, Fujimoto Y (1987) Deficiency of membrane-bound dipeptidyl aminopeptidase IV in a certain rat strain. Experientia 43:400-401

34. Pederson RA, Kieffer TJ, Pauly R, Kofod H, Kwong J, McIntosh $\mathrm{CH}$ (1996) The enteroinsular axis in dipeptidyl peptidase IVnegative rats. Metabolism 45:1335-1341

35. Ahren B, Hughes TE (2005) Inhibition of dipeptidyl peptidase-4 augments insulin secretion in response to exogenously administered glucagon-like peptide-1, glucose-dependent insulinotropic polypeptide, pituitary adenylate cyclase-activating polypeptide, and gastrin-releasing peptide in mice. Endocrinology 146:2055-2059

36. Flentke GR, Munoz E, Huber BT, Plaut AG, Kettner CA, Bachovchin WW (1991) Inhibition of dipeptidyl aminopeptidase IV (DP IV) by Xaa-boroPro dipeptides and use of these inhibitors to examine the role of DP IV in T-cell function. Proc Natl Acad Sci USA 88:1556-1559

37. Kubota T, Iizuka H, Bachovchin WW, Stollar BD (1994) Dipeptidyl peptidase IV (DP IV) activity in serum and on lymphocytes of MRL/Mp-lpr/lpr mice correlates with disease onset. Clin Exp Immunol 96:292-296

38. Hansen L, Deacon CF, Orskov C, Holst JJ (1999) Glucagon-like peptide-1-(7-36)amide is transformed to glucagon-like peptide-1(9-36)amide by dipeptidyl peptidase IV in the capillaries supplying the L cells of the porcine intestine. Endocrinology 140:5356-5363

39. Turton MD, O'Shea D, Gunn I (1996) A role for glucagon-like peptide-1 in the central regulation of feeding. Nature 379:69-72

40. Baggio LL, Huang Q, Brown TJ, Drucker DJ (2004) Oxyntomodulin and glucagon-like peptide-1 differentially regulate murine food intake and energy expenditure. Gastroenterology 127:546-558

41. Talsania T, Anini Y, Siu S, Drucker DJ, Brubaker PL (2005) Peripheral exendin-4 and peptide YY 3-36 synergistically reduce food intake through different mechanisms in mice. Endocrinology 146:3748-3756

42. Pospisilik JA, Stafford SG, Demuth HU (2002) Long-term treatment with the dipeptidyl peptidase IV inhibitor P32/98 causes sustained improvements in glucose tolerance, insulin sensitivity, hyperinsulinemia, and beta-cell glucose responsiveness in VDF ( $f a / f a)$ Zucker rats. Diabetes 51:943-950

43. Ahren B, Gomis R, Standl E, Mills D, Schweizer A (2004) Twelve- and 52-week efficacy of the dipeptidyl peptidase IV inhibitor LAF237 in metformin-treated patients with type 2 diabetes. Diabetes Care 27:2874-2880

44. Zander M, Christiansen A, Madsbad S, Holst JJ (2004) Additive effects of glucagon-like peptide 1 and pioglitazone in patients with type 2 diabetes. Diabetes Care 27:1910-1914

45. Poon T, Nelson P, Shen L et al (2005) Exenatide improves glycemic control and reduces body weight in subjects with type 2 diabetes: a dose-ranging study. Diabetes Technol Ther $7: 467-477$ 